\title{
Porta aperta: spazio riservato a ogni commento pertinente o impertinente
}

\section{A cura di Marco Lombardi $e$ Giovambattista Virga}

Questo spazio editoriale è dedicato ai commenti di chi è interessato a esprimere il proprio punto di vista sui temi, di volta in volta, trattati nei Supplementi. L'augurio è quello di poter ospitare la voce di nefrologi, infermieri, tecnici di nefrologia, dialisi e trapianto ma anche di medicina generale o altre specialità.

Sempre all'indirizzo dello stesso tema e con il permesso degli autori, vengono pubblicati anche alcuni commenti-scelti, apparsi sulla mailing-list Socisin, diretta da Giovambattista Virga e Piergiuseppe Delfino. Ci preme infine ricordare che quanto pubblicato di seguito, rispecchia solamente l'opinione personale degli autori che vi hanno partecipato.

\section{Come rispondere alla crescente domanda di dialisi?}

\author{
Marco Lombardi \\ U.O. di Nefrologia e Dialisi, Ospedale S.M. Annunziata, Azienda USL, Firenze
}

uasi quarantamila persone in Italia, sopravvivono a gravi forme di nefropatia cronica grazie ai trattamenti sostitutivi della funzione renale (1).

Ciò investe crescenti problematiche, non solamente sanitarie e assistenziali ma anche economiche e organizzative. Tali problematiche impattano sulla vita e sulle risorse dei pazienti e dei loro congiunti, che sono chiamati a un sostanziale sostegno e impegno, ma, in ultima analisi, anche su tutti gli utenti della sanità, perché le risorse disponibili non sono certamente infinite.

La necessità di ripristinare il vincolo di bilancio anche nel comparto sanitario, ha stimolato l'introduzione dell'aziendalizzazione nelle strutture sanitarie e l'attivazione d'altre forme concorrenziali, sia pur regolate. Tuttociò ha dato inizio, nella seconda metà degli anni ' 90 , alla percezione del "value for money", anche tra gli operatori della sanità pubblica (2).

Così, oggi, gli addetti ai lavori si trovano in questa si- tuazione: da un lato sono chiamati a contemperare le esigenze di contenimento e razionalizzazione della spesa sanitaria, dall'altro devono garantire la qualità necessaria delle cure per il benessere del paziente e dei suoi congiunti. Tuttociò cercando il vantaggio della propria azienda, in un'ottica di sana concorrenza (2).

Questa situazione sta producendo una nuova sperequazione tra dinamiche socio-demografiche e economicofinanziarie, la quale impone a tutti la necessità di ripensare agli attuali modelli assistenziali (2).

In particolare, nell'ambiente nefrologico, è sempre più sentita la questione di "Come rispondere alla crescente domanda di dialisi?"

La propagandata e incipiente riduzione delle risorse sanitarie, infatti, impone la necessità di cercare dei criteri sui quali poter fissare le priorità d'allocazione, tantoché provocatoriamente potrebbe tornar alla mente quel quesito che ha inquietato chi ha vissuto l'epoca pionieristica della dialisi " $\grave{E}$ giusto dializzare tutti?" 


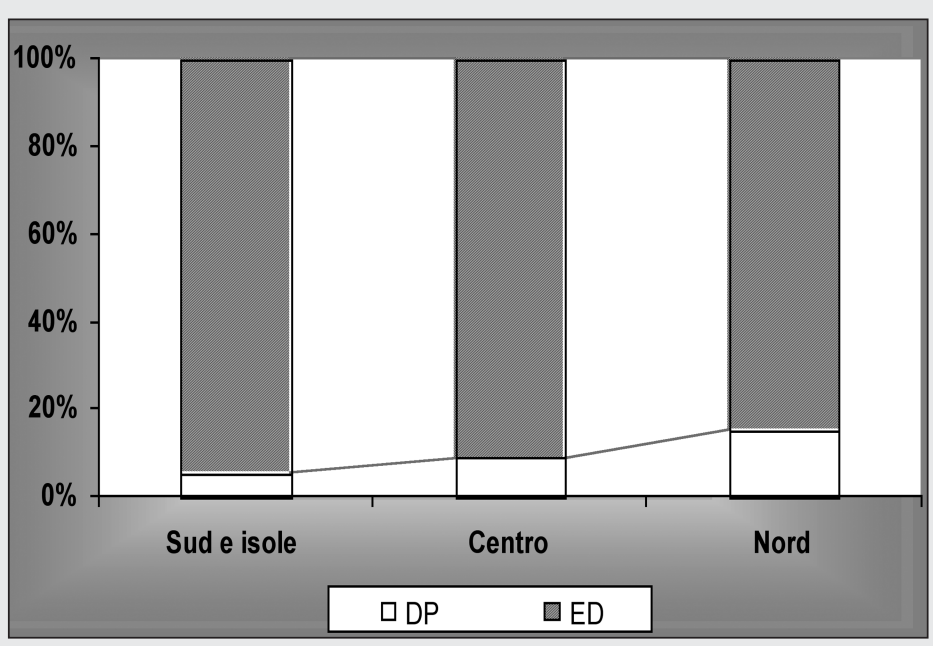

Fig. 1 - Diffusione dei due principali trattamenti in Italia.

Certamente sì, trovando però altre soluzioni a questa problematica.

\section{Il razionamento}

Un primo tipo di risposta potrebbe esser cercato nel razionamento (3). Ma non è corretto riconoscere al razionamento alcun ruolo né alcuna forma organizzativa o tantomeno risolutiva, poiché il Sistema Sanitario Nazionale, preposto alla tutela della salute dei cittadini, non può prevedere al suo interno nessuna forma d'inequità. E comunque noto a tutti che in sanità sono tuttora operanti dei meccanismi di "razionamento occulto", alludo alle liste d'attesa (le liste di trapianto renale, a esempio) la cui lunghezza è un'indubbia forma di razionamento occulto; o ancor peggio alla differenza territoriale dei servizi offerti, differenza di qualità e quantità, il cui esempio eclatante lo si può trovare nell'iniqua diffusione della dialisi peritoneale tra le Regioni italiane (4), (Fig. 1).

Viceversa, in alcuni Paesi anglosassoni si ritiene possibile gestire la priorità d'allocazione delle risorse implementando il razionamento $(3)$, cosa molto più difficile da proporre nel nostro Paese. A esempio, ove tra gli inglesi v'è tendenza ad accettare l'età come criterio discriminante all'accesso alle prestazioni, ciò non trova corrispondenza tra gli italiani (Fig. 2), che vedono una possibile e più equa soluzione nella ricerca di uno sfruttamento più efficiente delle risorse sanitarie.

Lo stesso studio del CENSIS (3) riporta i risultati di un'indagine dove un campione rappresentativo d'Italiani ha affermato che le risorse sanitarie non devono essere allocate in base all'età o ad altre discriminanti come, a esempio, precedenti comportamenti dannosi per la salute, e ove l'opinione dei cosiddetti testimoni privilegiati (responsabili di Asl, di Assessorati regionali alla Salute e d'altri osservatori politici), sui possibili potenziali scenari di riallocazione delle risorse è risultata ad-

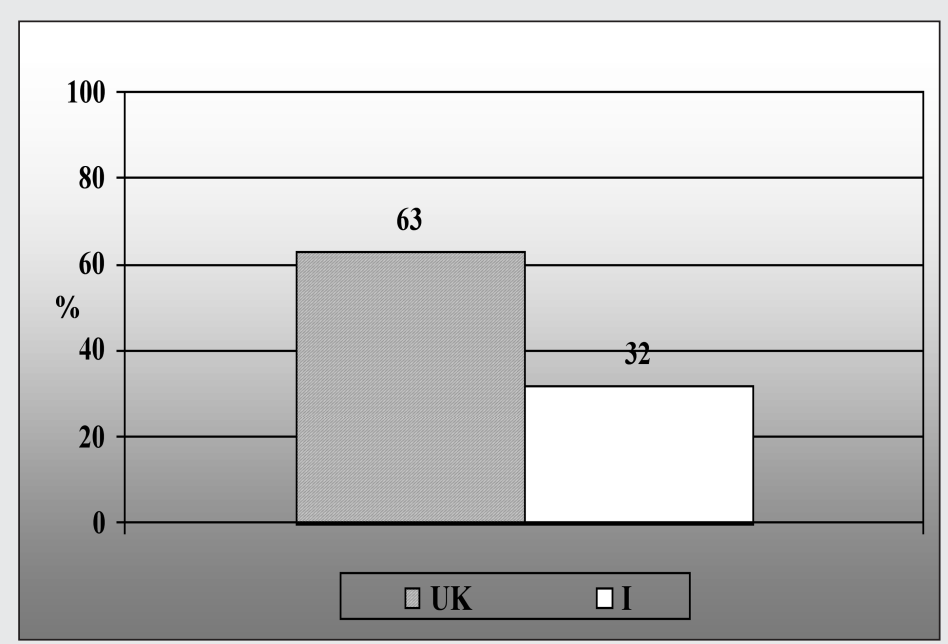

Fig. 2 - Percentuale d'accettazione dell'età come criterio discriminante all'accesso alle prestazioni sanitarie.

dirittura ancor più "orientata". ...Sembra infatti prevalere l'idea, che i potenziali processi ridistributivi debbano esser orientati verso un modello di offerta che trasferisca il baricentro dell'assistenza fuori dall'ospedale, sul territorio.

Così a livello dei servizi Asl, il 95\% degli intervistati ha affermato che la riallocazione delle risorse dovrebbe potenziare i servizi per gli anziani, il 91\% opta per un potenziamento dell' assistenza domiciliare, 1' $88 \%$ per quello dei day hospital e viceversa le risorse impiegate per l'assistenza ospedaliera dovrebbero esser ridotte per il $59 \%$ degli interpellati (3), (Fig. 3). A livello di spesa sanitaria, sempre secondo gli intervistati, la riallocazione delle risorse dovrebbe premiare e potenziare la prevenzione e l'assistenza extraospedaliera, mentre dovrebbe ridurre sia la spesa ospedaliera che quella per l'assistenza farmaceutica (3), (Fig. 4).

\section{Conviene l'autosufficienza?}

Ma è un altro studio del CENSIS quello che meglio riporta l'attuale possibile soluzione al problema della "crescente domanda di dialisi". Nel volume "Conviene l'autosufficienza?" (1), vengono infatti illustrati i molteplici vantaggi ottenibili dall'integrazione dei trattamenti dialitici e dalla riconsiderazione degli stessi in autosufficienza dalle strutture ospedaliere (naturalmente ove possibile).

In questo studio si afferma che al di là dell'indiscussa superiorità del trapianto renale per efficacia, efficienza e qualità di vita, le altre due tecniche sostitutive la funzione renale, ovvero l'emodialisi e la dialisi peritoneale, dimostrano pari efficacia, almeno nei primi 5 anni di somministrazione.

Oltre a ciò si afferma che - in questo ben definito arco di tempo - la DP può avere degli altri vantaggi. Alludo alla sua maggior capacità a mantenere la funzionalità renale residua, importante soprattutto nei pazienti che 


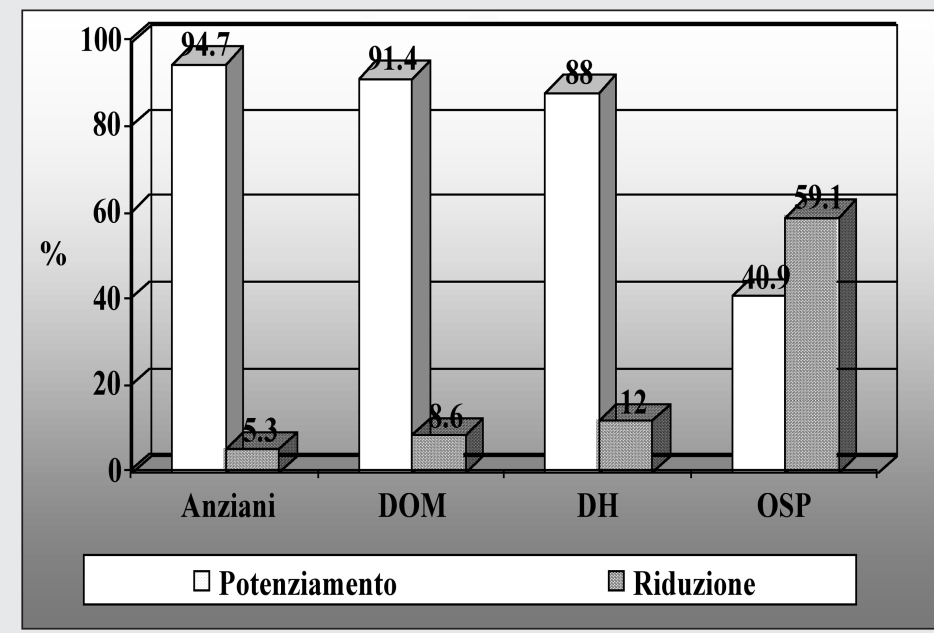

Fig. 3 - Possibili scenari di una riallocazione delle risorse nei servizi Asl, secondo l'opinione di testimoni privilegiati.

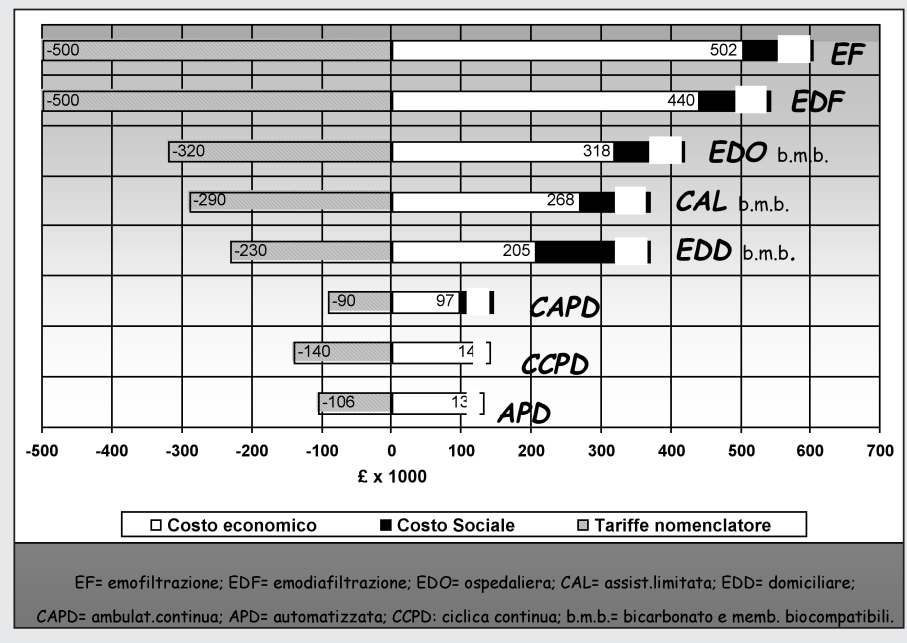

Fig. 5 - Costi delle principali metodiche dialitiche e rimborsi da tariffa ministeriale.

arriveranno a breve termine al trapianto renale; a quelle peculiarità che ne fanno una metodica quasi d'elezione in particolari tipologie di pazienti (età pediatrica, diabetici e gravi cardiovasculopatici); ma anche a quei vantaggi d'ordine economico: la dialisi peritoneale determina costi globali inferiori rispetto alle metodiche extracorporee.

Infatti, considerando il costo pieno come la somma tra costo sociale e costo economico (quest'ultimo costituito da varie voci: costo del personale, dei servizi alberghieri e generali, di manutenzione e ammortamento, dei materiali ecc.), si evidenzia come le tecniche peritoneali automatizzate risultino le più convenienti, seguite dalle altre forme di dialisi domiciliare, e come il confronto con le tariffe ministeriali risulti pressoché penalizzante (1), (Fig. 5). Anche analizzando i costi sostenuti dal Servizio Sanitario Nazionale - non per singolo trattamento ma per paziente per anno - risultano vantaggiose le tecniche domiciliari (1), (Fig. 6).

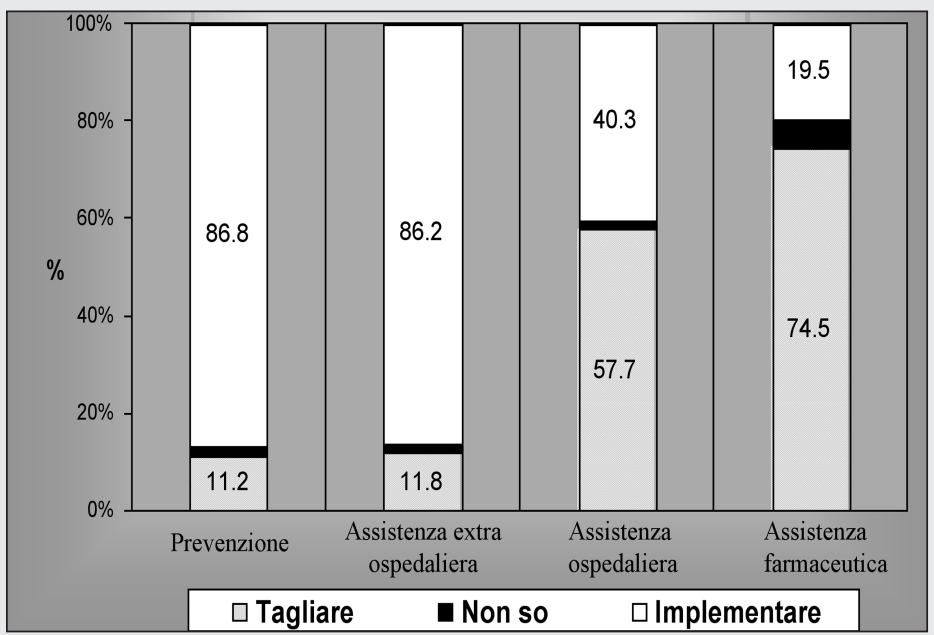

Fig. 4 - Orientamenti prevalenti in relazione a possibili scenari di riallocazione delle risorse a livello di spesa sanitaria, secondo l'opinione di testimoni privilegiati.

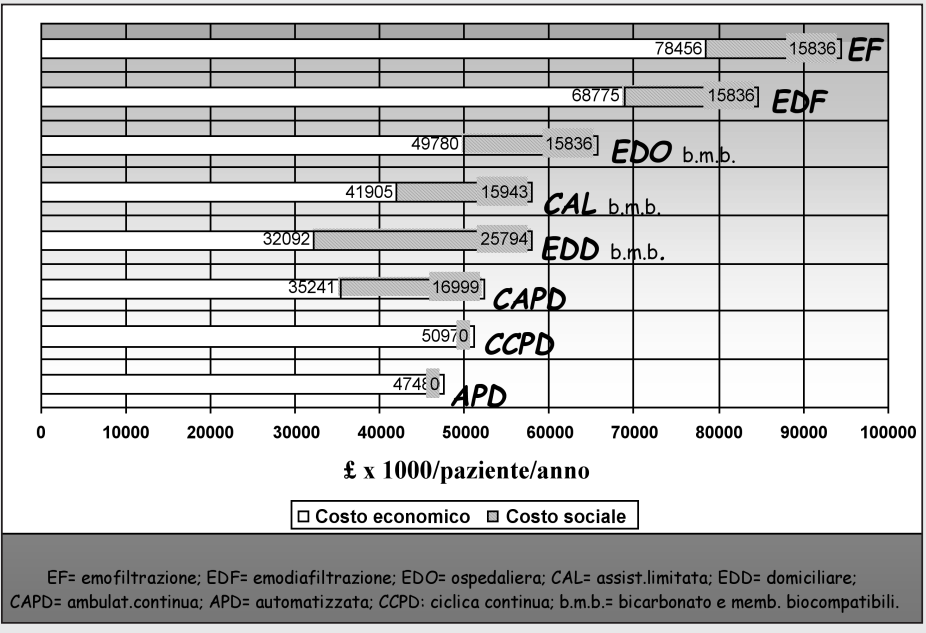

Fig. 6 - Costi della dialisi per paziente per anno.

Ma evidentemente al di la di tutti gli avvisi e inviti (più o meno allarmati) a contenere le spese, la comunità nefrologica non ha ritenuto di dover considerare il vantaggio insito nel maggior bilanciamento tra le metodiche dialitiche, tentando di modificare il tipo di trattamento sostitutivo iniziale.

Ciò è chiaramente espresso nei dati europei del 1995, quando su 100 uremici cronici terminali approdati al trattamento sostitutivo, 81 entravano in ED, 18 in DP e 1 solo accedeva al cosiddetto trapianto-pre-emptive, e tale approccio non era dissimile da quello che avveniva in Ialia (5), (Fig. 7).

Quali dunque i fattori, che hanno determinato e determinano una scelta di trattamento così sbilanciata e che rallentano la diffusione dell'integrazione dei trattamenti?

In estrema sintesi, secondo lo studio del CENSIS i fattori principali debbono esser ricercati nella:

a politica finanziaria e di rimborso sia per le aziende che per i medici; 


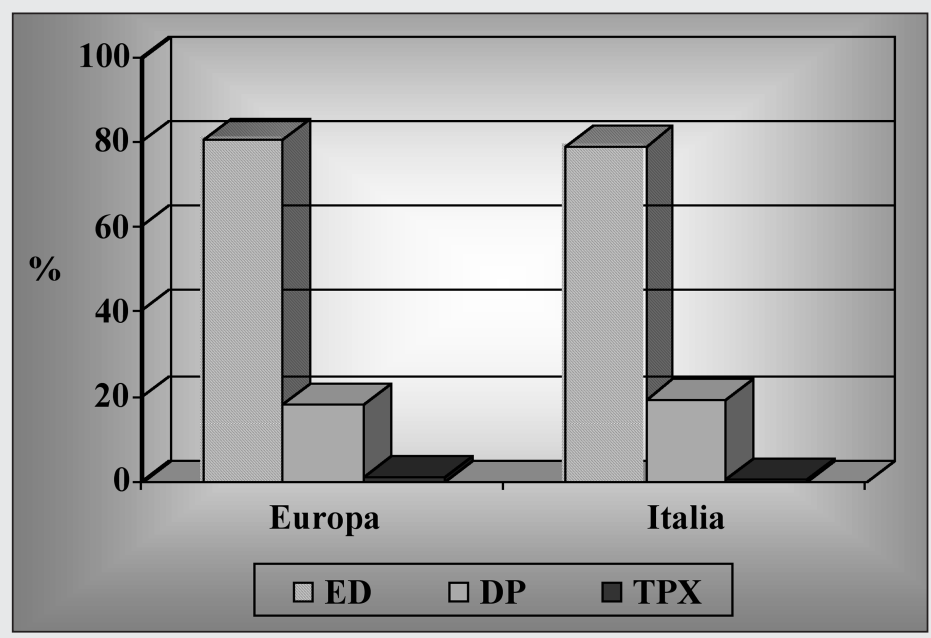

Fig. 7 - Differente uso dei trattamenti sostitutivi nel 1995.

a valutazione clinica derivata dal know-how e dal background del personale medico;

a possibilità d'offerta, essenzialmente dovuta alla numerosità delle strutture sul territorio e - particolarmente in Italia - al tipo di struttura (pubblica o privata);

a passaggio d'informazione, ovvero il grado di coinvolgimento nella decisione della scelta di trattamento, che i medici accettano di lasciare agli attori principali: i pazienti.

Prendere in considerazione e discutere le possibili cause e i rimedi a una così scarsa volontà a cercar d'integrare i trattamenti dialitici, esula dalla trattazione di quest'articolo, come esula cercare di rispondere al perché di una sempre più negletta considerazione ai trattamenti in autosufficienza dalle strutture ospedaliere.

Basti qui dire che molti credono che un'attuale e attuabile buona soluzione alla crescente domanda di dialisi risieda proprio nell'integrazione dei trattamenti dialitici e in una loro riconsiderazione anche in autosufficienza dalle strutture ospedaliere.

A suffragio di quanto sopra scritto, mi pregerò d'utilizzare i pareri di alcuni nostri colleghi, che hanno accettato d'esprimersi in proposito su due pubblicazioni che di recente hanno trattato l'argomento $(4,6)$.

\section{Hanno scritto...}

Al cospetto di una popolazione che cambia, negli anni ' 60 pochi pazienti selezionati, negli anni ' 70 molti giovani senza problemi extra-uremici, negli anni ' 80 sempre più anziani con problemi extra-uremici e negli anni '90 praticamente nessuna controindicazione esistente alla dialisi, è paradossale che la DP a fronte dei suoi tipici vantaggi, abbia avuto una così scarsa penetrazione (7). Può esser facilmente ipotizzato, dunque, lo scenario con il quale dovremo confrontarci in un prossimo futuro, ove fattori etici e deontologici impediranno di rifiutare il trattamento per motivi anagrafici, sociali o economici;
Fase uremica precoce (early referral): educazione alla dialisi domiciliare

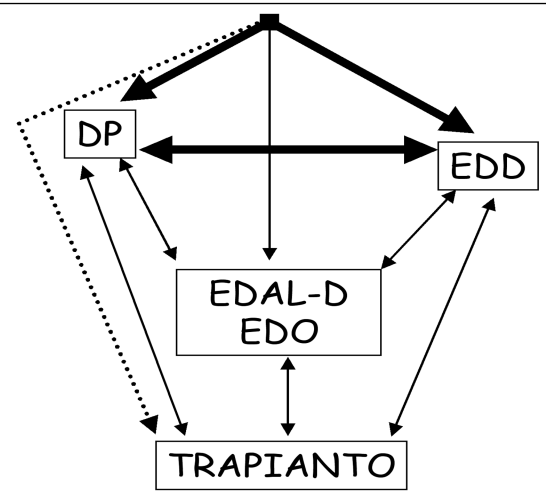

Fig. 8 - Trattamento sostitutivo a partire dalla dialisi domiciliare integrata.

la "dialisi per tutti" produrrà la necessità di ridurre i costi della dialisi, con spostamento verso nuove opzioni, come trattamenti meno costosi o in service a esempio, o preferenza per i trattamenti extraospedalieri. Si renderà necessaria la creazione di servizi sul territorio che risparmino le strutture ospedaliere, con maggior coinvolgimento di pazienti, familiari e servizi sociali, per approdare al risparmio sul personale più costoso, quello sanitario (7).

Ma altri cambiamenti s'imporranno prima che sia possibile pervenire a tal stato di cose, come stabilire a livello nazionale, la percentuale di pazienti in trattamento dialitico peritoneale per ogni Centro, favorire l'addestramento, anche a domicilio dei pazienti, creare sul territorio una rete di Centri in cui effettuare la dialisi automatizzata notturna e garantire ai pazienti un assegno mensile tipo "assegno di accompagnamento" (8).

Al contempo sarà necessario cominciare a combattere le diffidenze. Diffidenze da parte degli staff nei confronti di una tecnica nuova per il Centro e foriera di nuove "grane", diffidenza da parte dei pazienti, non informati correttamente o addirittura maliziosamente male informati (9), smettendo d'accettare che la DP non sia presente in tutti i Centri dialisi degni di tale nome, come parte integrante di un approccio completamente integrato al trattamento sostitutivo, perché anche da noi, come in altri Paesi europei, la garanzia del trattamento per tutti passa attraverso una via preferenziale verso la DP come trattamento iniziale (9).

La realtà però è assai diversa; purtroppo ancora troppo spesso non si considera che è più importante la sopravvivenza globale del paziente alla sopravvivenza nella singola metodica (10). In questo modo non si dà risposta alle richieste che ogni paziente pone al nefrologo e cioè di garantirgli la migliore sopravvivenza con l'utilizzo di tutte le metodiche disponibili nel rispetto della miglior qualità di vita (10).

L'introduzione di un budget annuo/ per paziente trattato, uguale su tutto il territorio nazionale potrebbe cambiare 
le cose? Applicando le tariffe indicate dal Ministero della Salute, il SSN rimborserebbe una quota budgetaria per paziente/anno, suscettibile di riduzione se si decidesse di programmare la maggiore penetrazione di una metodica a più basso costo rispetto a una più costosa (10). Sull'esempio di quanto accaduto in Canada, ove l'implementazione dei CAL ha visto una riduzione della DP sino all'introduzione governativa della quota capitaria (11). Ciò potrebbe risolvere il fatto che molti Centri privati a fronte dell'ormai nota uguaglianza tra le metodiche nei primi 5 anni di trattamento non hanno convenienza a far DP per la non remunerabilità del rimborso relativo, offerto dal SSN, in confronto a quello offerto per l'emodialisi (12).

D'altronde un movente più diffuso di quanto si creda a non far peritoneale- è dovuto al desiderio di chi dirige d'ottenere un maggior potere contrattuale e una maggiore visibilità nei confronti del sistema sanitario (13).

Ma la dialisi domiciliare o in autosufficienza dalle strutture ospedaliere vuol dire anche - in una percentuale tra il 2 e il 5\% - emodialisi domiciliare.

Recentemente in una pubblicazione su NDT è apparsa una sorta di classifica dei costi dei trattamenti ove il costo più alto è dovuto all'emodialisi nei Centri ospedalieri, poco inferiore quella nei Centri ad assistenza limitata e ancora minore quella per la EDD e la CAPD (14).

Ma se il contesto economico è cambiato, in questi ultimi quindici anni quello sociale non è cambiato più di tanto quando si pensa al concetto espresso dal Professor Vercellone ovvero "che nel proprio domicilio dializzano individui, non pazienti" (15). La scuola piemontese ancor oggi lascia intravedere la speranza che l'EDD torni in auge con la realizzazione della "one button machine" che esegua automaticamente priming, seduta dialitica in base alle esigenze metaboliche del paziente, reinfusione, lavaggio e disinfezione di linee e monitor ovviando, con il loro riutilizzo alla preparazione per la dialisi del giorno seguente (dialisi breve e giornaliera) (16).

Ma già oggi è una realtà la dialisi quotidiana domiciliare che è in grado di limitare grandemente l'interferenza sulla normale vita socio-lavorativa di paziente e partner grazie al programma di sedute ultrabrevi, inseribili negli spazi morti della giornata o nelle ore notturne; di migliorare drammaticamente le condizioni cliniche con tecnologie innovative ma che sono già una realtà dietro l'angolo, presto alla portata di tutti, come kit linee-filtro preassemblati, lavaggio e disinfezione di circuito e monitor automatizzati, riutilizzo del circuito extracorporeo, telemonitoraggio on-line della seduta e dei livelli di stoccaggio e dei consumi, miniaturizzazione delle apparecchiature ecc. (17).

A conclusione di quanto scritto da illustri colleghi sull'EDD, valga quanto affermato dal Prof. D'Amico e dal Professor Maiorca: solo un rifiuto preconcetto, legato a ignoranza o pigrizia mentale e una convenienza economica possono motivare la condanna acritica dell'EDD (18) e che tutte le metodiche vanno viste come tratta- menti che si integrano: il primo trattamento migliore è quello che piace di più al paziente, possibilmente a basso costo, quindi possibilmente fatto a domicilio (19).

\section{Conclusioni}

Una conclusione propositiva potrebbe esser quella di un modello da adottare con l'intento sia di rispondere alla crescente domanda di dialisi sia di cercar di soddisfare sempre di più le esigenze di quella parte di pazienti che sono in grado di raggiungere una riabilitazione e una qualità di vita "pressoché normali", con un programma di trattamento sostitutivo a partenza dalla dialisi domiciliare integrata (Fig. 8).

Tutti i pazienti precocemente inseriti nei cosiddetti ambulatori di pre-dialisi, ove giudicati idonei a un trattamento domiciliare, peritoneale o emodialitico, andrebbero "educati" ai trattamenti deospedalizzati, ovvero alla dialisi domiciliare. Una volta arrivati in utile prossimità del trattamento prescelto (al momento idoneo al confezionamento dell' accesso dialitico, catetere peritoneale, fistola A-V ecc.) i pazienti giudicati idonei - nel rispetto delle loro preferenze - dovranno iniziare il training. Una politica dei costi oltreché della qualità di vita consiglierà, comunque, il trattamento sostitutivo preferito dal paziente, ma ove possibile di dialisi peritoneale. Il programma di consolidamento educazionale alla dialisi a domicilio dovrà quindi proseguire anche durante il periodo di trattamento sostitutivo vero e proprio, mediante audit reiterati con il personale di dialisi, cosicché in caso di drop-out il paziente potrà essere shiftato - in prima battuta - verso l'EDD. Nei casi in cui non sia possibile un trattamento domiciliare, il paziente inizierà/verrà shiftato secondo le sue stesse preferenze verso un programma di dialisi, in prima scelta ad assistenza limitata/decentrata, e, in seconda scelta, ospedaliera.

Se il paziente è stato adeguatamente educato alla dialisi a domicilio, in caso di fallimento di trapianto - sempre che non sussistano controindicazioni di sorta e sempre nel rispetto della sua volontà - il rientro in trattamento sostitutivo dialitico sarà preferenziale per la DP o l'EDD.

Questo lavoro è stato presentato al Convegno su "L'insufficienza renale cronica: dalla terapia conservativa alla sostitutiva”. Arezzo, 8 Febbraio 2002. 


\section{BIBLIOGRAFIA}

1. CENSIS. Conviene l'autosufficienza? Un percorso verso l'integrazione dei trattamenti dialitici. Franco Angeli Ed. Milano, 2001.

2.

http://www.censis.it/censis/ricerche/1999/21051999.htm. Il mercato Sanitario in Europa. Il ruolo dell'ospedalità privata.

3.

http://www.censis.it/censis/ricerche/1999/22061999.htm. È giusto curare tutti? Criteri per il razionamento in sanità.

4. Dialisi peritoneale: dialisi marginale? A cura di Marco Lombardi. Giornale di Tecniche Nefrologiche \& Dialitiche 2001; 13 (Suppl. 1): S3-66.

5. Berthoux F, Jones E, Geller R, et al. Epidemiological data of treated end-stage renal failure in the European Union (EU) during the year 1995: report of the European Renal Association Registry and the National Registries. Nephrol Dial Transplant 1999; 23: 32-42.

6. Ha ancora senso fare emodialisi domiciliare nel 2001? A cura di Marco Lombardi. Giornale di Tecniche Nefrologiche \& Dialitiche 2001; 13 (Supp1. 2): S3-74.

7. Catizone L. Coraggio si riparte! Giornale di Tecniche Nefrologiche \& Dialitiche. 2001; 13 (Supp1. 1): S16-8.

8. Brunori G. Dialisi peritoneale in Italia: adeguatamente utilizzata o è possibile un ulteriore incremento? Giornale di Tecniche Nefrologiche \& Dialitiche 2001; 13 (Suppl. 1): S10-1.

9. Buoncristiani U. I motivi per i quali in Italia la percentuale dei pazienti in DP rimane stabilmente bassa. Giornale di Tecniche Nefrologiche \& Dialitiche 2001; 13 (Suppl. 1): S12-3.

10. Corciulo R. La dialisi peritoneale: perché dialisi marginale? Giornale di Tecniche Nefrologiche \& Dialitiche 2001; 13 (Supp1. 1): S24-6.

11. Pacitti A, Maffei S, Fenoglio R. La bassa prevalenza in dialisi peritoneale: realtà italiana ed estera a confronto. Giornale di Tecniche Nefrologiche \& Dialitiche 2001; 13 (Suppl. 2): S63-8.

12. La Greca G. Perché la dialisi peritoneale in Italia ha una bassa penetrazione. Giornale di Tecniche Nefrologiche \& Dialitiche. 2001; 13 (Suppl. 1): S39-42.

13. Viglino G. Troppo poca DP in Italia. Giornale di Tecniche Nefrologiche \& Dialitiche 2001; 13 (Suppl. 1): S59-60.
14. De Vecchi AF, Dratwa M, Wiedemann ME. Healthcare systems and end-stage renal disease (ESRD) therapies - an international review: cost and reimbursement/funding of ESRD therapies. Nephrol Dial Trasplant 1999; 14 (Suppl): S31-41.

15. Vercellone A. Riflessioni sull'emodialisi domiciliare (2a parte). Dialisi Oggi 1985; 5: 37-42.

16. Alloatti S. L'emodialisi domiciliare. Giornale di Tecniche Nefrologiche \& Dialitiche 2001; 13 (Suppl. 2): S9-11.

17. Buoncristiani U. Emodialisi domiciliare: cause della crisi e basi per la ripresa. Giornale di Tecniche Nefrologiche \& Dialitiche 2001; 13 (Suppl. 2): S17-9.

18. D'Amico G. Il trattamento emodialitico oggi. Giornale di Tecniche Nefrologiche \& Dialitiche. 2001; 13 (Supp1. 2): S23-4.

19. Maiorca R. Ha ancora senso fare oggi EDD. Giornale di Tecniche Nefrologiche \& Dialitiche. 2001; 13 (Supp1. 2): S34. 